\title{
EINE SPÄTANTIKE TOGABÜSTE AUS BALÇOVA
}

Im Jahre 1965 wurde bei Balçova (Türkei) eine Büste entdeckt, die bislang nicht ausführlich publiziert worden ist und hier vorgestellt werden soll ${ }^{1}$. Sie stammt aus dem stilistischen Umfeld der spätantiken Porträtgruppe um den Kopf des sog. Eutropius aus Ephesos. Von den Vertretern dieser Gruppe sind nur Porträtköpfe bekannt. Das Einmalige an unserem Porträt ist die vollständig erhaltene und in einem gearbeitete Büste, die mit der Toga in ihrer spätantiken Form dargestellt ist. Die Gruppe um Eutropius wird nach rein kunsthistorisch stilistischen Kriterien zumeist in die zweite Hälfte des 5. Jahrhunderts datiert. Anhand der Gewandung der neuen Büste soll die Frage der Datierung diskutiert werden.

\section{Die Büste}

Bereits im Jahr 1965 war in Balçova, südwestlich von Izmir, beim Graben eines modernen Brunnens im sog. Agamemnon-Thermalbad ${ }^{2}$ die Büste eines Mannes ${ }^{3}$ mittleren Alters gefunden worden (Abb. 1. 2) . Von der Büste, die aus einem weißlichgrauen, grobkörnigen Marmor ${ }^{5}$ ge-

1 Der Generaldirektion für Monumente und Museen bin ich zu Dank für die Erlaubnis verpflichtet, diese Büste publizieren zu dürfen (Schreiben des stellvertretenden Generaldirektors A. Karaoğlu vom 29. Mai 2000). M. Aurenhammer danke ich vor allem für die kritische Durchsicht des Manuskripts sowie für die hilfreiche Unterstützung, die Publikationserlaubnis zu erlangen. Dank sei auch an das Museum von Izmir und seine Mitarbeiterinnen und Mitarbeiter gerichtet, die uns immer großzügig unterstützen. H. Teoman sei an dieser Stelle herzlichst für seine ständige Hilfsbereitschaft und seinen unermüdlichen Einsatz vor Ort in den Depots des Museums gedankt. Für die Erstellung der Photos danke ich N. Gail (ÖAI Wien). - Abkürzungen und Zitierweise folgen den ÖJh 69, 2000, 357 ff. (http://www.oeai.at/publik/autoren.html). Darüber hinaus gelten folgende Abkürzungen:

Goette $(1990)=$ H. R. Goette, Studien zu römischen Togadarstellungen (1990).

İnan - Rosenbaum (1969) = J. İnan - E. Rosenbaum, Roman and Early Byzantine Portrait Sculpture (1966).

İnan - Alföldi-Rosenbaum $(1979)=$ J. İnan - E. Alföldi-Rosenbaum, Römische und frühbyzantinische Porträtplastik aus der Türkei (1979).

IvE = Inschriften griechischer Städte aus Kleinasien 11, 1 (1979) - 17, 4 (1984), Ephesos (IvE Ia-VII 2).

Kollwitz (1941) = J. Kollwitz, Oströmische Plastik der theodosianischen Zeit (1941).

Oberleitner (1959) = W. Oberleitner, Fragment eines spätantiken Porträtkopfes aus Ephesos, ÖJh 44, 1959, 83 ff. Oberleitner $(1964 / 65)=$ W. Oberleitner, Beiträge zur Geschichte der spätantiken Porträtplastik aus Ephesos, ÖJh 47, 1964/65, 5 ff.

Özgan - Stutzinger $(1985)=$ R. Özgan - D. Stutzinger, Untersuchungen zur Porträtplastik des 5. Jhs. n. Chr. anhand zweier neugefundener Porträts aus Stratonikeia, IstMitt 35, 1985, $237 \mathrm{ff}$.

2 Zur modernen Lokalität des Bades s. Y. Gül, Antik Kentler ${ }^{2}$ (1998) 46 f. 142. Hier verweist Y. Gül auf Tagungen im Museum von Izmir, die Balçova zum Inhalt hatten. Leider waren mir die Akten nicht zugänglich. - Über den Ort der heißen Quellen am Meer berichtet z. B. schon Strab. 14, 645, weiters sind sie bei Philostr., Heroikos 691, 20 erwähnt. Nach ihm befinden sie sich in der Nähe der Stadt Smyrna und werden von den Bewohnern der Stadt Agamemnon-Quellen genannt.

3 Izmir, Arkeoloji Müzesi Inv. 5246. Material: weißlichgrauer, grobkörniger Marmor. In Gelb-, Grün- und Brauntönen ist der Marmor verwittert. Maße: erh. GesamtH 0,59 m, BüstenB 0,46 m, KopfH 0,24 m, KopfT 0,19 m, GesichtsH $0,2 \mathrm{~m}$, erh. GesichtsB (an den Backenknochen) 0,13 m.

4 Die Angaben zu den Fundumständen sind entnommen aus: Gül (Anm. 2) Abb.-Teil (»1965 yılında Balçova Agamemnon 1lıcalarinde sıcak su kuyusu kazılırken ortaya çıkan bu büst İzmir Arkeoloji müzesindedir«).

5 Der Marmor wurde noch nicht naturwissenschaftlich untersucht. Die auf reiner Autopsie beruhende Beschreibung des Materials gestaltet sich wegen des schlechten Erhaltungszustands der Büste entsprechend schwierig. 
fertigt und in einem mit dem Porträtkopf gearbeitet wurde, fehlt heute lediglich der Büstenfuß $\beta^{6}$. Die Schulterpartie mit dem Brustkorb ist flach wiedergegeben, allein der Kopf tritt weit nach vorne gestreckt aus dieser Ebene hervor (Abb. 3. 4).

Durch die offensichtlich jahrhundertelange Lagerung im Wasser ist die Marmoroberfläche, vor allem an der linken Seite der Büste, stark korrodiert. Die linke Gesichtshälfte ist leider vollständig zerfressen, so daß sich die originale Oberfläche dort nicht erhalten und sich auch das Volumen der Büste stark verringert hat (Abb. 8). Hingegen sind einzelne Partien der rechten Gesichtshälfte in einem erstaunlich guten Erhaltungszustand (Abb. 7), abgesehen von einer dünnen Sinterschicht, die sich während der Lagerung bildete. Trotz des beklagenswerten Gesamtzustands der Büste hat sich der Eindruck einer ehemals äußerst sorgfältigen und feinen Arbeit erhalten.

Auf dem langgestreckten Hals ruht ein schmaler, länglicher Kopf(Abb. 5). Die Blickrichtung des Porträtierten geht streng nach vorne. Das langrechteckige Gesichtsfeld wird durch eine deutlich ausgeprägte Kieferpartie mit leicht vorspringendem, abgesetztem Kinn abgeschlossen. Am Porträtkopf fallen die hohe Stirn mit geradem Haarabschluß, die eingefallenen, schlaffen Wangen mit dominant hervortretenden Jochbeinen sowie die lange, ausgeprägte Nase mit dem schlanken Nasenrücken und der schmale Mund auf. Markant tritt die Augenpartie mit den sich stark wölbenden Augäpfeln in Erscheinung. Ob die Iris des rechten Auges - das wesentlich besser erhalten ist als das linke - durch eine halbkreisförmige Bohrung dargestellt war, kann nicht mehr entschieden werden. Der Augapfel selbst wird begrenzt von einem schmalen, scharfkantig abgesetzten Oberlid. Das schwere Unterlid ist hoch über den Augapfel gezogen und durch eine feine Kerbe von der Wangenpartie abgesetzt. Die Augenbrauen ziehen in einem weitgespannten Bogen über die Augen, sie scheinen direkt in die schmale Nasenwurzel überzugehen. Über den äußeren Augenwinkeln enden sie gratig und stehen damit im Kontrast zu den weichen, hängenden Orbitalen. Von dem äußeren Augenwinkel geht eine gekerbte horizontale Falte aus, unter der sich das Jochbein zu wölben beginnt. Die Schläfen sind 'eingezogen', darunter verbreitert sich das Gesicht stärker zu den Jochbeinen hin. Die schlaff hängenden Wangen werden durch tief eingeschnittene Nasolabialfalten begrenzt, die Nase verbreitert sich gleichmäßig zur Nasenspitze.

Der Dargestellte trägt einen leicht gekräuselten, kurzen Bart, der, vor den Ohren beginnend - dort erhöht wiedergegeben -, über die Wangen zum Kinn zieht (Abb. 7). Das Philtrum dürfte ausgespart geblieben sein. Der Bart ist mit feinen Kerben in das Inkarnat eingetieft.

Das durch feine Härchen gegliederte Haupthaar ist durch eine erhöht angedeutete Haarkappe gestaltet, die über den Schläfen ihr größtes Volumen erreicht (Abb. 5-8). Lange Haarsträhnen ziehen radial vom Scheitelmittelpunkt auf der Kalotte über den Kopf. Die Ausarbeitung der Haare am Hinterkopf kann wegen der starken Versinterung der Kopfrückseite nicht beobachtet werden. Das Haar reicht nicht weit in den Nacken und schließt mit einer leichten Rundung zu beiden Seiten hinter den Ohren ab; direkt vor den Ohren geht das Haupthaar in den Backenbart über. Am linken Ohr sind nur noch die groben Umrisse aufgrund der Verwitterung des Gesteins zu erkennen. Das rechte Ohr ist am oberen Ohrrand beschädigt, zusätzlich ist es stark versintert. Die Ohren sind schmal und langgezogen mit einem kleinen abgesetzten Ohrläppchen.

Die gesamte Gesichts- und Halsoberfläche ist von feinen Feilspuren überzogen. An der Büstenvorderseite ist die ursprüngliche Oberfläche kaum erhalten, jedoch sind an der linken Schulter partiell feine Raspelspuren zu sehen: die Oberfläche der Gewandung wurde also nicht restlos geglättet. Zwischen Armansatz und linker Brust hat sich eine weitere Stelle der Oberfläche erhalten.

\footnotetext{
${ }^{6}$ Auf der einzigen bislang bekannten Abbildung (Gül [Anm. 2] Abb.-Teil) ist ein Büstenfuß zu sehen. Dieser konnte im Museumsdepot von Izmir nicht aufgefunden werden.
} 

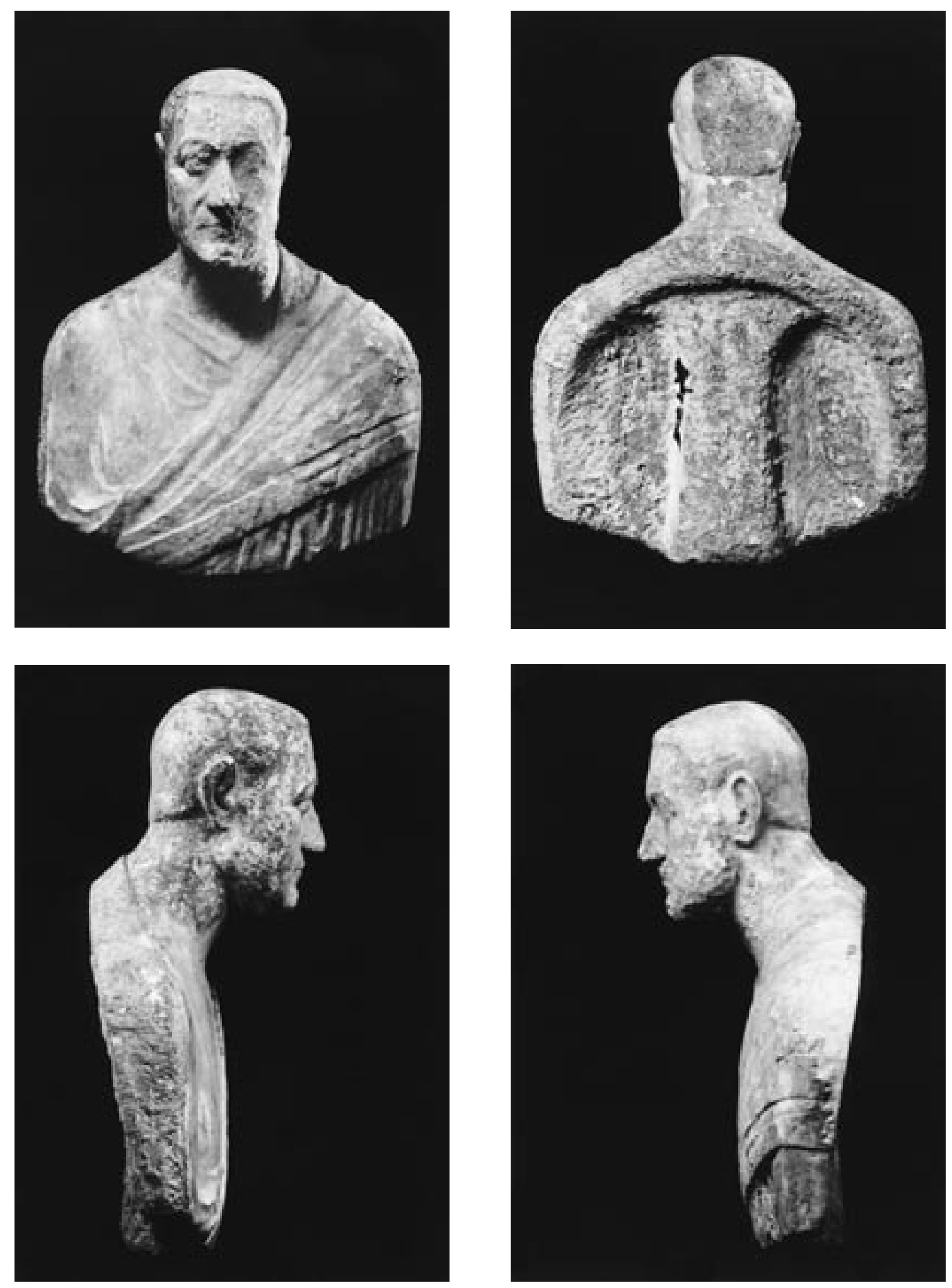

1-4 Büste aus Balçova. Izmir, Arkeoloji Müzesi Inv. 5246 
Die Büstenrückseite (Abb. 2) ist von einer starken Sinterschicht überzogen, trotzdem sind die Bearbeitungsspuren des Spitzmeißels noch deutlich auszumachen. Die Büstenstütze verbreitert sich leicht nach unten, ihr oberer Abschluß schwingt zu beiden Seiten bis zur Mitte der Schulter aus. Zu beiden Seiten der Stütze ist die Büste ausgehöhlt. Der Büstenrand ist von der Stütze abgesetzt, seine Unterseite ist mit dem Zahneisen geglättet. In der Mitte befindet sich ein Dübelloch mit dem Rest eines Metallstiftes. Der Büstenfuß war somit extra gearbeitet und montiert. Nach der Photographie bei Y. Gül ${ }^{7}$ war er profiliert und von zylindrischer Form mit einer runden Standfläche ${ }^{8}$.

Der Dargestellte trägt die Toga in ihrer spätantiken Form9. Sowohl in der Terminologie als auch in der Typologie beziehe ich mich im Folgenden auf H. R. Goette ${ }^{10}$. Er gliederte die Darstellung der Toga von ihrem Beginn in republikanischer Zeit bis in die Spätantike in fünf Typen, wobei er spätantike Togen auf Grund ihrer unterschiedlichen Umboformen ${ }^{11}$ in zwei Typen unterteilte: Typus D und Typus E. Typus D ist die Toga mit voll contabuliertem Umbo, Typus E jene mit flachem, gefächertem Umbo. Die Bekleidung unserer Büste war ursprünglich sehr flach gestaltet, die einzige markante Erhebung ist das an der rechten Seite herausgezogene Colobium. Auffällig an der Gewandung ist der schräge, steil hochziehende Umbo, der aufgefächert unter dem rechten Armansatz hervortritt und sich flach über der linken Schulter verbreitert. Unsere Büste wäre demnach Goettes Typus $E^{12}$ zuzurechnen. An der linken Brustseite unterhalb des Umbo befinden sich vertikale Falten, die sich von einer angedeuteten Bogenfalte an der rechten Seite unterscheiden.

Die Entstehungszeit der Büste kann also nach der Art der Gewanddrapierung in der Spätantike fixiert werden. Unter den bekannten spätantiken Porträts steht unser Porträt den Köpfen rund um 'Eutropius' aus Ephesos am nächsten.

7 Gül (Anm. 2) Abb.-Teil.

8 H 0,125 m; DübellochDm 0,01 m. Getrennt gearbeitete Büstenfüße konnten bereits für die Spätantike belegt werden, s. E. Alföldi-Rosenbaum, JbAC 15, 1972, 176.

9 Bezüglich der Forschungsgeschichte zur Toga in ihrer spätantiken Form s. J. Wilpert, Die Gewandung der Christen in den ersten Jahrhunderten. Vornehmlich nach den Katakomben-Malereien dargestellt (1898) 7 ff.; ders., Die Malerei der Katakomben Roms (1903). Von L. M. Wilson stammt die erste sehr ausführliche Besprechung der Toga in der Spätantike. Sie beschäftigte sich vor allem mit den Schnittmustern, die zu den unterschiedlichen Drapierungen der Toga führen (L. M. Wilson, The Roman Toga [1924] 89 ff.; dies., The clothing of the ancient Romans [1938]). Weitere grundlegende Arbeit leistete R. Delbrueck, Die Konsulardiptychen und verwandte Denkmäler (1929) 43 ff. Er beschäftigte sich besonders mit der Terminologie der einzelnen Togateile. Wilson und Delbrueck gingen bezüglich der Rekonstruktion der spätantiken Toga vorwiegend von den Darstellungen auf den Konsulardiptychen aus; rundplastische Beispiele waren in den 20er und 30er Jahren des 20. Jahrhunderts kaum bekannt. Eine für längere Zeit verbindliche Darstellung der Entwicklung der Toga stammt von F. W. Goethert in: RE VI A 2 (1937) 1651 ff. s. v. Toga. Die erste umfassende Vorstellung der rundplastischen Vertreter der spätantiken Togati und eine erste zeitliche Einordnung verdankt die Forschung Kollwitz (1941). Das Fundmaterial konnte seither speziell in Ephesos und Aphrodisias vermehrt werden. So publizierte Oberleitner $(1959 ; 1964 / 65)$ die Statue des sog. Stephanos aus Ephesos; im Anschluß daran erstellte er eine neue chronologische Reihe der spätantiken Porträtstatuen. Özgan - Stutzinger (1985) unternahmen dies mit Hilfe der Gruppe der Chlamydati.

10 Goette (1990) 3 ff. 59 ff.

11 Anders: H. Wrede, Gnomon 67, 1995, 544. Für ihn ist es nicht der Umbo, der die Brust quert, sondern der Balteus, auch sieht er »die linke Körperseite offen« und geht in Folge auf das Problem der tunica palmata und toga picta ein. Der Frage der Drapierung kann nach Wrede nur mit Hilfe der Rückseiten nachgegangen werden; weiters existiert für ihn nicht die Trennung in Typus D und E.

12 Goette (1990) 62 ff. 

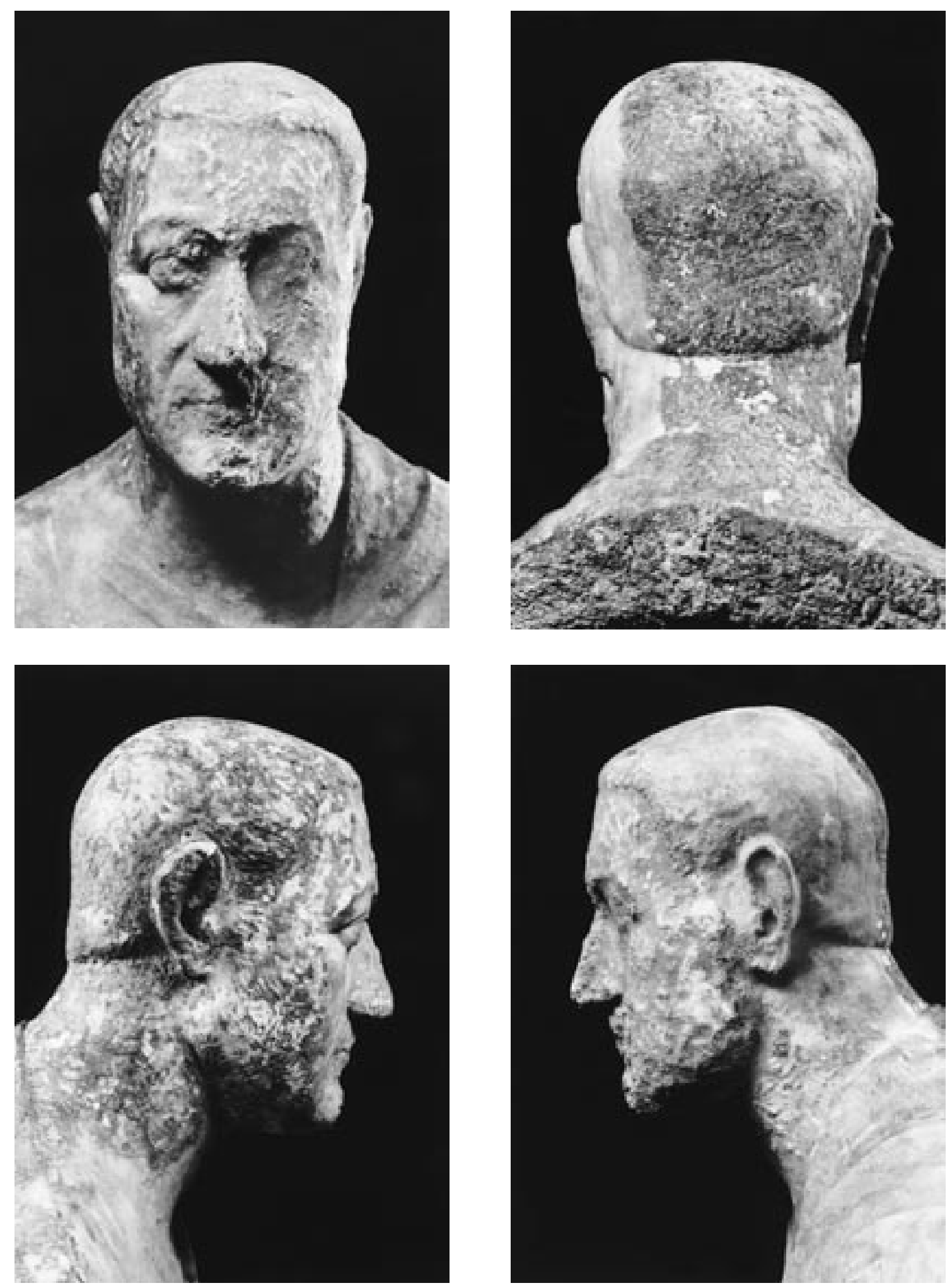

5-8 Kopf der Büste aus Balçova. Izmir, Arkeoloji Müzesi Inv. 5246 


\section{Die Eutropius-Gruppe}

Bereits G. Rodenwaldt ${ }^{13}$ stellte zwei Köpfe aus Ephesos, heute im Kunsthistorischen Museum in Wien, nebeneinander: den Kopf des 'Eutropius' ${ }^{14}$ und den 'Lockenkopf' ${ }^{15}$, welcher sich durch dichte Lockenangabe am Vorderkopf auszeichne; die Köpfe seien nicht voneinander zu trennen ${ }^{16}$. Rodenwaldt stellte sie in einen größeren Kreis von Porträts und sah Gemeinsamkeiten - vor allem in der Behandlung des Haares - mit dem 'Älteren Magistraten' ${ }^{\prime 17}$ aus Aphrodisias, heute im Archäologischen Museum in Istanbul, weiters in bezug auf die »einheitlich und rundlich weich geformte Masse« der Haare mit der Kaiserstatue aus Aphrodisias, dem 'Valentinian II. ${ }^{18}$, der ebenfalls in Istanbul aufbewahrt wird. Zeitlich setzte Rodenwaldt den Kopf des sog. Eutropius als spätestes Werk an ${ }^{19}$. H. P. L'Orange schloß sich in seiner Studie zur Geschichte des spätantiken Porträts dieser Meinung an, auch er sieht ein sehr enges Verhältnis zwischen 'Eutropius' und dem 'Lockenkopf'20.

W. Oberleitner wollte die beiden Porträts von derselben Künstlerhand, dem »Eutropiusmeister $\ll^{21}$, entstanden sehen. Zusätzlich postulierte er einen Werkstättenzusammenhang für weitere Porträts ${ }^{22}$, die dem des 'Eutropius' anzuschließen sind. J. İnan und E. Rosenbaum stellten noch ein Porträt zu dieser Gruppe, ein leider jetzt verschollenes Kopffragment mit der Fundortangabe »Agora von Izmir« ${ }^{23}$. Schließlich konnte M. Aurenhammer einen Neufund aus Ephesos vorstellen

13 G. Rodenwaldt, Griechische Porträts aus dem Ausgang der Antike, 76. BWPr (1919) 20 ff.

14 Zum 'Eutropius', Wien, Kunsthistorisches Museum, Antikensammlung Inv. I 880: R. v. Schneider, Ausstellung von Fundstücken aus Ephesos im Unteren Belvedere² (1916) 29 Nr. 33; Rodenwaldt (Anm. 13) 20. 26. 29 Nr. 13 Abb. 8. 9; H. P. L'Orange, Studien zur Geschichte des spätantiken Porträts (1933) 84 ff. 92.146 f. Nr. 115 Abb. 216. 217; F. Eichler, AnzWien 76, 1939, 5 ff. Abb. 1. 2. 6; Kollwitz (1941) 128 ff. Taf. 43; W. Alzinger, ÖJh 42, 1955, 41 Abb. 18; G. Egger, JbKuHistSamml 51, 1955, 24 ff. Abb. 14. 15; Oberleitner (1964/65) 16 ff. Abb. 9. 10; İnan - Rosenbaum (1966) 151 ff. Nr. 194 Taf. 181, 1. 2; H. v. Heintze in: Th. Kraus (Hrsg.), Das römische Weltreich, Propyläen Kunstgeschichte II (1967) 265; D. Metzler, AA 84, 1969, 202; H. G. Severin, Zur Portraitplastik des 5. Jhs. n. Chr. (1972) 108 ff. Nr. 22; R. Fleischer in: A. Bammer - R. Fleischer - D. Knibbe, Führer durch das Archäologische Museum in Selçuk-Ephesos (1973) 77; R. Noll, Vom Altertum zum Mittelalter, Kunsthistorisches Museum Wien. Katalog der Antikensammlung I² (1974) Kat. A 13 Abb. 5; S. Sande, ActaAArtHist 6, 1975, 74 ff.; İnan - Alföldi-Rosenbaum (1979) 33 ff. 186 mit Anm. 1; 188. 228. 232 ff. 243. 306 f.; K. Weitzmann (Hrsg.), Age of Spirituality (Ausstellungskat. New York, Metropolitan Museum of Art 1979) 58 Nr. 55; J. Meischner, BJb 181, 1981, 160 ff. Anm. 125. 131; D. Stutzinger in: Spätantike und frühes Christentum (Ausstellungskat. Liebieghaus, Museum alter Plastik, Frankfurt am Main 1983) 464 Nr. 70; M. Aurenhammer, ÖJh 54, 1983, Beibl. 141 ff.; J. Meischner, JdI 106, 1991, 385 ff. Taf. 86, 1; F. A. Bauer, Stadt, Platz und Denkmal in der Spätantike (1996) 278 f. Abb. 83 Taf. 30, 1; R. R. R. Smith, JRS 89, 1999, 185; M. Bergmann in: Aurea Roma. Dalla città pagana alla città cristiana (Ausstellungskat. Rom, Palazzo delle Esposizioni 2000) 241. 647 Kat. 355 mit Abb.; J. Meischner, Bildnisse der Spätantike. 193-500. Problemfelder. Die Privatporträts (2002) 111 f. 122 Abb. 369.

15 Wien, Kunsthistorisches Museum, Antikensammlung Inv. I 835. Rodenwaldt (Anm. 13) 21. 26 f. 28 f. Nr. 14 Abb. 10. 11; İnan - Rosenbaum (1966) 153 Nr. 195 Taf. 182, 1. 2 (mit älterer Lit.); Oberleitner (1964/65) 21 ff. Nr. 3 Abb. 11. 12; W. Oberleitner, AW 2/2, 1971, 37 f. Abb. 3; Severin (Anm. 14) 113 f. 179 f. Nr. 23; İnan - Alföldi-Rosenbaum (1979) 36. 187. 189. 243. 306 f.; Meischner (Anm. 14:1991) 386; Özgan - Stutzinger (1985) 272; Meischner (Anm. 14:2002) 123 Abb. 359. 360.

16 Severin (Anm. 14) 113 f. ist wegen der unterschiedlichen Physiognomie gegen die Miteinbeziehung des 'Lockenkopfes' in die Eutropiusgruppe.

17 Istanbul, Arkeoloji Müzeleri Inv. 2265; zuletzt Smith (Anm. 14) 176 f. Taf. 1, 3; 6, 2.

18 Istanbul, Arkeoloji Müzeleri Inv. 2264; zuletzt Smith (Anm. 14) 162 Abb. 4 Taf. 12, 1 f.

19 Rodenwaldt (Anm. 13) 26.

20 L'Orange (Anm. 14) 86.

21 Oberleitner (1964/65) 26.

22 1. Porträtkopf: Selçuk, Efes Müzesi Inv. 340. Oberleitner (1964/65) 16 ff. Nr. 1 Abb. 7. 8; 2. Porträtfragment: Selçuk, Efes Müzesi Inv. 182. Oberleitner (1964/65) 25 ff. Nr. 4 Abb. 13. 14. - Die Qualität würde an die beiden Köpfe in Wien nicht heranreichen, aber sie seien derselben Werkstatt zuzuordnen.

23 Zu dem Fragment: TürkAD 2, 1934, 232 Abb. 15; R. Naumann - S. Kantar, IstForsch 17, 1950, 103 f. Nr. 28 Taf. 39a-c; İnan - Rosenbaum (1966) 120 Nr. 134 Taf. 180, 1. 2: »The coiffure and the style link this portrait with that of >Eutropius in Vienna, the rendering of the beard constituting one of the major differences. The similarities are so close that it seems certain that the two heads are of the same date.«-Meischner (Anm. 14:1991) 389. 395. 
- einen männlichen Einsatzkopf -, der ebenso der Eutropius-Gruppe hinzuzufügen ist ${ }^{24}$. K. T. Erim erwog bei einem verschollenen Kopf aus den Hadriansthermen von Aphrodisias eine Zusammengehörigkeit mit den Porträts rund um 'Eutropius'. Von diesem Kopf existieren lediglich alte Photographien ${ }^{25}$; wegen der schlechten Qualität der Ablichtung ist die Zuweisung nicht sicher möglich.

Den Datierungsrahmen für den Porträtkopf des 'Eutropius' bildet in der Forschung das 5. Jahrhundert n. Chr. Es gelang jedoch noch nicht, die Gruppe um 'Eutropius' »durch absolute Kriterien ... sicher $[\mathrm{zu}]$ datieren, wie überhaupt die Stilabfolge der Porträts des 5. Jhs. vorwiegend nur durch Bezüge zwischen verschiedenen Porträtgruppen relativ festgesetzt werden kann. $\ll^{26} \mathrm{H}$. v. Heintze bevorzugte eine Frühdatierung des Kopfes des 'Eutropius' und sah ihn in der ersten Hälfte des 5. Jahrhunderts entstanden ${ }^{27}$. H. G. Severin ließ bei der Datierung des 'Eutropius' große Vorsicht walten ${ }^{28}$, könnte sich aber vorstellen, »daß sie ['Eutropius' und das Porträt des Kolosses von Barletta] noch in spättheodosianischer Zeit gearbeitet sind - insbesondere wenn die Regierung Markians (450-457) in diese Epoche einbezogen und eine anläßlich des Regierungswechsels veränderte Konzeption des Kaiserportraits in Rechnung gestellt wird. $\ll^{29}$

Der Großteil der Forschung schloß sich jedoch einer späteren Datierung an. J. Kollwitz zeigte - wie H. G. Severin - die Verbindungen zu dem Porträt des Kolosses von Barletta ${ }^{30}$ und, die Gestaltung der Augen betreffend, zu einem Kopf im Louvre ${ }^{31}$ auf: beide seien in ihrer Entstehung kurz vor dem Porträt des 'Eutropius' anzusetzen. Dadurch gelangte Kollwitz zu einer Datierung um die Jahrhundertmitte ${ }^{32}$. J. Meischner ${ }^{33}$ setzte den Kopf des Eutropius wegen des »hohe[n] Grad[es] seiner Abstraktion« an das Ende einer in frühtheodosianischer Zeit beginnenden Entwicklungreihe und gelangte dadurch mit der Datierung in das sechste Jahrzehnt des 5. Jahrhunderts n. Chr. Den »Eutropius-Typus« verband sie zuletzt, wie bereits Kollwitz, mit dem Kopf Valentinians III. ${ }^{34}$ in Paris ${ }^{35}$. H. P. L'Orange stellte den Kopf des 'Eutropius' im Vergleich mit Darstellungen auf Diptychen ${ }^{36}$ in die zweite Hälfte des 5. Jahrhunderts n. Chr. ${ }^{37}$. J. İnan und E. Rosenbaum sahen ihn im dritten Viertel des 5. Jahrhunderts n. Chr. entstanden ${ }^{38}$, S. Sande siedelte 'Eutropius' ebenso in der zweiten Hälfte des 5. Jahrhunderts n. Chr. an ${ }^{39}$. W. Oberleitner versuchte in Hinblick auf 'Eutropius', den er »auf dem Gipfel des künstlerischen Schaffens seines Meisters entstanden $\aleph^{40}$ sah, die weiteren Porträts der Eutropius-Gruppe chrono-

399. 407 Taf. 88, 1; Kollwitz (1941) 130 hob auch schon die Gemeinsamkeiten des 'Eutropius’ mit dem Fragment aus Izmir hervor.

24 Selçuk, Efes Müzesi Inv. 4/32/83: H. Vetters, AnzWien 120, 1983, 120 Taf. 14a. b; Aurenhammer (Anm. 14) 140 ff. Abb. 14. 15; dies. in: N. Bonacasa - G. Rizza (Hrsg.), Ritratto Ufficiale Internazionale sul Ritratto Romano. Roma 1984, Quaderni de 'La Ricerca Scientifica' 116, 1988, 128 ff.; Meischner (Anm. 14:1991) 386 f. 391 ff. Taf. 86, 2; Meischner (Anm. 14:2002) Abb. 368.

${ }_{25}$ K. T. Erim, AJA 71, 1967, 237 f. Taf. 69, 14-16; İnan - Alföldi-Rosenbaum (1979) 236 Anm. 4 zu Nr. 207.

26 Aurenhammer (Anm. 14) 143.

27 v. Heintze (Anm. 14) 265 Nr. 336.

28 Severin (Anm. 14) $108 \mathrm{ff}$.

29 Ebenda 113.

30 s. dazu R. Stichel, Die römische Kaiserstatue am Ausgang der Antike. Untersuchungen zum plastischen Kaiserporträt seit Valentinian I. (364-375 n. Chr.) (1982) 61 ff. Taf. 30. 31. Stichel sieht in der Bronzestatue von Barletta die Darstellung des Kaisers Zenon.

31 s. R. Delbrueck, Spätantike Kaiserporträts von Constantinus Magnus bis zum Ende des Westreiches. Studien zur spätantiken Kunstgeschichte (1933) 215 Taf. 112; zuletzt: K. de Kersauson, Musée du Louvre. Catalogue des portraits romains II (1996) 538 f. Nr. 257 mit Abb.

32 Kollwitz (1941) $129 \mathrm{f}$.

33 Meischner (Anm. 14:1991) $385 \mathrm{f}$.

34 Kersauson (Anm. 31) Nr. 257.

35 Meischner (Anm. 14:2002) 124.

36 Hier vor allem das Diptychon des Boethius (487): Delbrueck (Anm. 9) $103 \mathrm{ff}$.

37 L'Orange (Anm. 14) 89 ff.

38 İnan - Rosenbaum (1966) $151 \mathrm{f}$.

39 Sande (Anm. 14) 74.

40 Oberleitner (1964/65) 24. 
logisch zu reihen. So seien der 'Lockenkopf' 41 und der Oberteil eines Porträtkopfes ${ }^{42}$ zuerst entstanden, nämlich um die Mitte des 5. Jahrhunderts n. Chr. Der Kopf des 'Eutropius' müsse danach in der zweiten Hälfte des 5. Jahrhunderts entstanden sein. Nicht von der Hand des »Eutropiusmeisters« sei ein Porträt eines bärtigen Mannes, der von 'Eutropius' jedoch »weitgehend beeinflußt ist $\aleph^{43}$ und somit zeitgleich bzw. kurz danach gefertigt wurde.

Wie unsicher die zeitliche Einordnung und die Interpretation des 'Eutropius' selbst sind, wird einmal mehr durch die problematische Angabe der Fundumstände verdeutlicht. Die Büste des 'Eutropius' war unweit einer Konsole, die auf ihrer Vorderseite eine Inschrift ${ }^{44}$ trägt, in Ephesos am nördlichen Ende der Marmorstraße gefunden worden. F. Eichler stellte sie gemeinsam mit der Beschreibung der Fundumstände ausführlich vor ${ }^{45}$, mußte sich jedoch eingestehen, da $ß$ »ein zwingender Beweis für die Kombination von Kopf und Inschrift ... nicht zu erbringen [ist], die Argumente beruhen hauptsächlich auf der Fundnähe und der sicheren Zugehörigkeit des Kopfes zu einer Büste einerseits, der Bettung für eine solche auf dem Kragstein mit der Inschrift anderseits und die Größenverhältnisse sind möglich; alles andere wird man der Vermutung günstig finden oder je nachdem als bloße Konstruktion ablehnen. $\aleph^{46}$ Eichlers Argument für eine Zusammengehörigkeit von Konsole und Inschrift liegt im »eigenartigen formalen Bau des Kopfes, dessen prismatische Grundform drei gleichwertige Ansichten ergibt ... dem die Straße entlang Gehenden bot sich eine der beiden Profilansichten dar, dem vor dem Denkmal Stehenbleibenden und die Inschrift Lesenden die Frontalansicht. $\ll^{47}$

So sind nicht einmal bei dem bekannten Kopf des 'Eutropius' die Fundumstände so eindeutig, daß die Zusammengehörigkeit von Büste und Konsole gesichert ist. Auch wenn Büste und Konsole zusammengehören sollten, kann die Nennung eines Eutropius zu einer genaueren Datierung nichts beitragen, da dieser Name im 4. und 5. Jahrhundert häufig belegt ist ${ }^{48}$. C. Foss sieht in der Namensnennung der Inschrift einen Proconsul von Asia des späten 5. Jahrhunderts n. Chr., wobei sich seine Datierung an die kunsthistorisch zeitliche Stellung des Porträtkopfes anlehnt ${ }^{49}$.

\section{Die Stellung der Büste aus Balçova innerhalb der Eutropius-Gruppe}

Für den Vergleich unserer Büste aus Balçova mit Bildnissen der Eutropius-Gruppe sollen das Porträt des 'Eutropius' ${ }^{50}$ selbst, der 'Lockenkopf' ${ }^{51}$ und jener Einsatzkopf ${ }^{52}$, der 1982 in Ephesos gefunden wurde, herangezogen werden. Weitere Köpfe der Eutropius-Gruppe werden im Folgenden vernachlässigt. Der Kopf von der Agora von Izmir $^{53}$ dürfte, was seine rechte Nebenseite betrifft, dem 1982 in Ephesos gefundenen Einsatzkopf sehr nahestehen. Das Fragment eines weiteren Lockenkopfes ${ }^{54}$ kann wegen der unterschiedlichen Frisur in unserem Falle außer acht

41 S. o. Anm. 15

42 s. o. Anm. 22.

${ }^{43}$ Oberleitner (1964/65) 27.

44 IvE 1304.

45 Eichler (Anm. 14) 5 ff.; s. zusätzlich: R. Heberdey, ÖJh 10, 1907, Beibl. 71 ff.

46 Eichler (Anm. 14) 12.

47 Ebenda 11.

48 RE VI 1 (1907) 1520 ff. s. v. Eutropius (Seeck - Jülicher); C. Foss, Stephanus, Proconsul of Asia, and Related Statues, in: Okeanos. Essays presented to I. Ševčenko, Harvard Ukrainian Studies 7, 1983, 202 f.; D. Feissel, Antiquité Tardive 6, 1998, 98 Anm. 65.

49 C. Foss, Ephesus after Antiquity: A late Antique, Byzantine and Turkish City (1979) 61 ff.; ders. (Anm. 48) $202 \mathrm{f}$.

50 s. o. Anm. 14.

51 s. o. Anm. 15.

52 s. o. Anm. 24.

53 s. o. Anm. 23.

54 s. o. Anm. 22. 
gelassen werden. Auch jener stark zerstörte Kopf ${ }^{55}$, den W. Oberleitner als eine Nachahmung des 'Eutropius' empfand, soll für den Vergleich unberücksichtigt bleiben. Vernachlässigt wird er in der Untersuchung nicht nur wegen seines Haares - besonders seiner Stirnfrisur -, das rundum abgeschlagen ist, sondern auch wegen der stark überzeichneten Details des Gesichtfeldes.

Die Gemeinsamkeiten unseres Porträts mit jenen der Eutropius-Gruppe sind offensichtlich. So treffen wir auch an der Büste in Izmir die in die Länge gezogene Kopfform an, die starke Akzentuierung der Augenpartie, die tief eingeschnittenen Nasolabialfalten, die Verbreiterung des Gesichtsfeldes im Bereich der Jochbeine und den zusammengekniffenen, dünnlippigen Mund. Was jedoch die Haar- und Barttracht unserer Büste betrifft, stoßen wir auf die größten Unterschiede zu den anderen Vertretern der Gruppe (abgesehen von kleineren Details der Physiognomie).

Der Kopf der Büste aus Balçova zeichnet sich durch eine enganliegende Haarkappe aus, die in ihrem hinteren Abschluß - wie bei dem 'Lockenkopf' - im Nacken sehr weit oben endet. Das Haar dürfte auch bei unserem Kopf, wie bei allen anderen Porträts, in langen, leicht gewellten Strähnen von einem Scheitelpunkt auf der Kalotte ausgehen. Man vermißt die Darstellung einer Nackenrolle, wie sie bei dem 1982 in Ephesos gefundenen Einsatzkopf gut ausgebildet zu erkennen ist. Bei 'Eutropius' hingegen und dem 'Lockenkopf' ist sie wesentlich flacher ausgebildet. Die das Gesicht umrahmenden Haare sind ähnlich den Haaren des 'Eutropius' angeordnet, doch reichen bei 'Eutropius' die gesträhnten Haare tiefer in die Stirn und wölben sich stärker seitlich der Schläfen. Mit dem Einsatzkopf aus Ephesos verglichen, ist die Frisur der Büste aus Balçova insgesamt kürzer. Der Einsatzkopf trägt das Haar länger, es bauscht sich rechts und links der Schläfen auf. Zusätzlich hat er eine sehr hohe Stirn, die durch Geheimratsecken verstärkt wird, in der Stirnmitte liegt lediglich eine nach links gestrichene Locke. In der Wiedergabe des Bartes wird ein großer Unterschied zu 'Eutropius' und dem Einsatzkopf einerseits und zu dem 'Lockenkopf' andererseits offensichtlich. Wie bereits beschrieben, ist bei unserer Büste der Bart durch kurze, geschwungene Härchen angegeben. Die drei weiteren Köpfe der Eutropius-Gruppe tragen die Bärte länger, sie sind durch leicht geschwungene, lange Haare charakterisiert. Der 'Lockenkopf' trägt einen voluminösen, das Gesicht umrahmenden Bart.

Unterschiede sind natürlich auch in der Physiognomie festzustellen, wobei aber unser Kopf viele Gemeinsamkeiten mit dem Einsatzkopf aus Ephesos zeigt. Dies betrifft den Schwung der Brauen, die stark hängenden Orbitale und die Darstellung des Mundes, der breiter ist als bei den anderen Köpfen und dessen Mundwinkel nicht so stark hinunter gezogen sind. Ein wichtiger gemeinsamer Erkennungsfaktor bei der Eutropius-Gruppe ist die Darstellung der Ohren: So sind alle rechten Ohren fast gleich gestaltet, ebenso alle linken. Dies kann aber bei der Büste aus Balçova wegen ihres schlechten Erhaltungszustands nicht mehr festgestellt werden.

An den Vertretern dieser spätantiken Porträtgruppe sind die aufgerauhten Gesichts- und Halsoberflächen charakteristisch und deutlich zu sehen. Auch diese Eigenart verbindet unseren Kopf vor allem mit dem Einsatzkopf aus Ephesos. Der Gesamteindruck unseres Kopfes ist wesentlich naturalistischer als bei 'Eutropius' oder dem 'Lockenkopf', wodurch er dem Einsatzkopf von $1982 \mathrm{sehr}$ nahesteht.

Die Frage nach der zeitlichen Eingrenzung des Porträts aus Balçova ist noch unbeantwortet. Einmalig an diesem neuen Porträt der Eutropius-Gruppe ist die vollständig erhaltene und in einem gearbeitete Büste. Vielleicht kann die Darstellung der Toga im Vergleich mit anderen Togadarstellungen der Spätantike einen Hinweis auf die zeitliche Stellung geben.

\footnotetext{
55 s. o. Anm. 22.
} 


\section{Die Toga-Büste}

Uns ist eine Reihe von Büsten mit der Darstellung der Toga in ihrer spätantiken Form bekannt; aus Aphrodisias ist bislang leider nur ein Fragment einer Büste publiziert, obwohl wir von dort eine Vielzahl von Gewandstatuen aus der Spätantike kennen ${ }^{56}$. Aus Griechenland ist die sehr feine und sorgfältig ausgearbeitete Büste eines bärtigen Mannes in Athen ${ }^{57} \mathrm{zu}$ nennen, deren Kopf mit der Büste in einem gearbeitet ist. Daß es sich ursprünglich um eine Büste gehandelt hatte, wurde von J. Kollwitz angezweifelt ${ }^{58}$, er interpretierte sie als aus einer Statue umgearbeitet ${ }^{59}$. Die Büste wird allgemein in das zweite Viertel des 5. Jahrhunderts datiert ${ }^{60}$.

Alle weiteren Togabüsten bzw. der größte Teil der spätantiken Büsten stammt von dem Fundort Ephesos. Hier sind vor allem zwei äußerst qualitätvolle, seit langem aus der Literatur bekannte Büsten zu nennen ${ }^{61}$. Zuletzt wurden sie von R. Özgan und D. Stutzinger an das Ende des 5. Jahrhunderts gesetzt ${ }^{62}$, S. Sande datierte ihre Entstehung erst in das 6. Jahrhundert ${ }^{63}$. W. Oberleitner nahm für beide Büsten dieselbe Werkstatt an, wobei die Gemeinsamkeiten besonders in der Augenbildung zu beobachten wären: »... engverwandt sind die schematisch und linear gearbeiteten Büsten. Das gleiche gilt von deren Hinterseiten, sowohl was die Form betrifft als auch hinsichtlich der verwendeten Werkzeuge. ${ }^{64}$ Beide Büsten besitzen eine sich nach unten zu leicht verbreiternde Mittelstütze, wobei der Büstenrand nicht von dieser abgesetzt ist, sondern in derselben Ebene abschließt. Die Oberfläche ist mit einem groben Zahneisen bearbeitet. Der Unterschied zu der Rückseite der Büste aus Balçova (Abb. 2) ist augenfällig und würde die Herkunft der Büsten aus unterschiedlichen Werkstätten bestätigen ${ }^{65}$.

Aus Ephesos stammt eine weitere Büste mit angearbeitetem Büstenfuß, die von H. R. Goette nur mit einer Photographie veröffentlicht wurde ${ }^{66}$ : Kopf und Büste waren in einem gearbeitet, die Bruchstelle des Halses ist im Durchmesser auffällig groß. Der Brustkorb - der Büstenausschnitt reicht bis unterhalb der Brust - ist flach wiedergegeben. Die Schultern schließen nicht in einer Ebene ab, die rechte Schulter liegt wesentlich tiefer als die linke. Die Büste ist im unteren

\footnotetext{
56 Aphrodisias Inv. 70-600 A-B: R. R. R. Smith - Ch. Ratté, AJA 101, 1997, 17 Abb. 14: »... a type previously unattested at Aphrodisias ...«.

57 Athen, Nationalmuseum Inv. 423: L. v. Sybel, Katalog der Skulpturen zu Athen (1881) Nr. 690; L'Orange (Anm. 14) 89 Anm. 3; Kollwitz (1941) 91. 104. 125 ff. Nr. 18 Taf. 41, 1. 2; Oberleitner (1959) 88. 94 f.; Severin (Anm. 14) 61 ff. Nr. 13; E. Alföldi-Rosenbaum, JbAC 1972, 176 Taf. 14c; Foss (Anm. 48) 214 mit Anm. 78; Meischner (Anm. 14:1991) 386 Taf. 87, 1; Goette (1990) 147 E 14; 153 L 77 Taf. 58, 1; Meischner (Anm. 14: 2002) Abb. 367.

58 Kollwitz (1941) 91.

59 Anders: Alföldi-Rosenbaum (Anm. 57) 176.

${ }^{60}$ Kollwitz (1941) 91 (»430/40«); Oberleitner (1959) 95 (»440«); Severin (Anm. 14) 81 (»1. Hälfte 5. Jh.«).

61 1. Büste: Selçuk, Efes Müzesi Inv. 850. 743. 1956/255. 1958/2. Oberleitner (1959) 87 f. 93 f. 97 Abb. 63. 64; İnan - Rosenbaum (1966) 156 f. Nr. 201 Taf. 184, 2; 185, 3. 4; Fleischer (Anm. 14) 83 f.; İnan - Alföldi-Rosenbaum (1979) 27 ff. 31. 237. 287 Anm. 1; 300. 306; Sande (Anm. 14) 90 f. Abb. 36-38; Meischner (Anm. 14:1981) 161 ff. Abb. 8; Foss (Anm. 48) 199 mit Anm. 10; Özgan - Stutzinger (1985) 253 f. 271 ff. Taf. 53, 4; S. Sande, AA 1992, 602 mit Anm. 2; Goette (1990) 70. 147 E 15; 153. 178; Bauer (Anm. 14) 425. - 2. Büste: Selçuk, Efes Müzesi Inv. 2034. H. Vetters, ÖJh 49, 1968-71, Beibl. Grabungen 1969, 9 Abb. 2; ders., AnzWien 107, 1970, 4 Nr. 10 Taf. 1a; Alföldi-Rosenbaum (Anm. 57) 176 Taf. 14b (Rückseite); Sande (Anm. 14) 90 ff. Abb. 39. 40; İnan - Alföldi-Rosenbaum (1979) 189 f. Nr. 156 Taf. 267; Foss (Anm. 48) 199 mit Anm. 10; Özgan - Stutzinger (1985) 272 Anm. 113; S. Sande, AA 1992, 602 mit Anm. 2; Goette (1990) 70. 147 E16. L79; M. Aurenhammer, ÖJh 68, 1999, Jahresbericht 1998, 36 Abb. 16.

62 Özgan - Stutzinger (1985) 253 f. 271 ff.

63 Sande (Anm. 14) $90 \mathrm{f}$.

64 İnan - Alföldi-Rosenbaum (1979) $189 \mathrm{f}$.

65 Über den enormen Erkenntniswert von Büstenrückseiten für Werkstattzusammengehörigkeiten s. zuletzt K. Fittschen, Eine Werkstatt attischer Porträtbildhauer im 2. Jh. n. Chr., in: Ch. Reusser (Hrsg.), Griechenland in der Kaiserzeit. Neue Funde und Forschungen zu Skulptur, Architektur und Topographie. Kolloquium zum sechzigsten Geburtstag von Prof. Dietrich Willers, Bern, 12.-13. Juni 1998 (2001) 71 ff.

${ }^{66}$ Selçuk, Efes Müzesi, o. Inv.: s. Goette (1990) 147 unter E16 Taf. 58, 3.
} 
Bereich nicht fertig ausgearbeitet. Der Büstenfuß ist nur roh angelegt, die Hohlkehle erst mit dem Spitzmeißel vorbereitet, die Leisten darüber und darunter wurden vorläufig mit dem Zahneisen bearbeitet. Vielleicht sekundär ist eine nahezu vertikale Kerbe, die zwischen dem linken Oberarm und der Brust verläuft. Natürlich könnte es sich dabei auch um einen Versuch handeln, den Oberarm zu akzentuieren. Die Rückseite der Büste, grob mit dem Spitzmeißel angelegt, ist nicht fertiggestellt.

In einem anderen Exemplar aus Ephesos läßt sich eine Büste nachweisen, die aus einer Statue umgearbeitet wurde. Es handelt sich dabei um den Oberkörpertorso eines Togatus, der in sekundärer Verwendung als Büste dienen sollte ${ }^{67}$. Die Umarbeitung war geplant, es kam aber nicht zur fertigen Ausführung, der Arbeitsprozeß wurde eingestellt. Eine durchgehende Reihe von Spitzmeißelansätzen, die alle zur Halsmitte gerichtet sind, hat sich um den Halsrand erhalten, so daß der Eindruck entsteht, als wäre der ursprünglich in einem gearbeitete Kopf absichtlich abgeschlagen worden, um eventuell eine Höhlung oder zumindest eine Ansatzfläche für einen Einsatzkopf zu schaffen. Die Rückseite dieses Statuenfragments ist nur grob vorbereitet, um es seiner anderen Bestimmung zuzuführen. Auch läßt sich schon der untere geplante Abschluß der Büste ausmachen: Sie sollte in einem sanft gerundeten Bogen enden.

Der Rest der Bekleidung, der an der 'Eutropius-Büste' erhalten ist, legt für die zu rekonstruierende Büste die Darstellung der Toga nahe ${ }^{68}$. Weit zieht der Umbo an der linken Halsseite hinauf. An der Halskante des Einsatzkopfes aus dem Jahr 1982 findet sich an der linken Halsseite von dem Rest der Tunika ausgehend ein breiterer, aufgerauhter und erhabener Streifen, der bis unter den Nacken reicht und an der rechten Halsseite ausdünnt; dort ist der Hals auch tiefer hinuntergezogen. Die zu erwartende Bekleidung ist auch hier die Toga, die an der linken Halsseite durch die Anlage des Umbo höher hinaufreicht.

Die Büste aus Balçova läßt sich, was die Darstellungsweise ihrer Gewandung betrifft, kaum mit einer der bekannten Togabüsten vergleichen. Auffallend an der Büste ist der schräg über die Brust nach oben ziehende Umbo, der bereits unter dem rechten Ansatz des Oberarmes breit hervortritt. Der Umbo umhüllt den Brustkorb aber nicht vollständig - wie wir dies zum Beispiel bei der Statue des Stephanos ${ }^{69}$ finden -, sondern scheint locker zur linken Schulter gelegt zu sein. Direkt unter dem Ansatz des Umbo auf der rechten Seite befindet sich der Beginn einer Bogenfalte, diese wird in der Brustmitte von vertikalen Falten abgelöst. Gemessen an anderen spätantiken Togati ist es durchaus legitim, hier von einer 'naturalistischen' Faltenwiedergabe gerade dieser Partie unterhalb des Umbo zu sprechen ${ }^{70}$. Eine entfernte Ähnlichkeit scheint mit der Büste

${ }^{67}$ Selçuk, Efes Müzesi, o. Inv. (unpubl.): erh. H 0,51 m; grobkörniger, hellgrau gemaserter Marmor. H. R. Goette publizierte ein Photo: Goette (1990) 147 unter E16 Taf. 58, 4.

68 Für Foss (Anm. 48) 203 (»Traces of drapery represented on the neck and shoulder of the bust indicate that it was clothed in a toga. This example, too, suggests the obvious and inevitable conclusion that the proconsul of Asia wore the toga.«) ist der Kopf des 'Eutropius' mit dem Büstenansatz neben der Statue des 'Stephanos' ein weiterer Beleg dafür, daß sich ein Proconsul in der Toga darstellen ließ. Zu diesem Problem s. weiter M. Horster, Antiquité Tardive 6, 1998, 48: »Es ist aufgrund der bisher bekannten Statuen sehr wahrscheinlich, daß es keine spezifische Ikonographie für Statuen von Statthaltern und wahrscheinlich ebenfalls nicht von einer Reihe anderer Amtsträger in der Spätantike gegeben hat, die über die allgemeinen Standesabzeichen der Senatoren und insbesondere der Amtsträger einer bestimmten Rangstufe hinausgehen.« Auch Smith (Anm. 14) 179 ff. sieht in der Toga nicht nur die Gewandung des Proconsul. - Zur Statue des 'Stephanos', Selçuk, Efes Müzesi Inv. 1402. 1956/83: F. Miltner, AnzWien 94, 1957, 23; ders., TürkAD 7, 1957, 13 Taf. 8, 8; ders., ÖJh 44, 1959, Beibl. 280 f. Abb. 137; Oberleitner (1959) 86. 90.92 f. 97.99 f. Abb. 60-62; İnan - Rosenbaum (1966) 157 f. Nr. 202 Taf. 178, 4; 186, 4. 5; H. Vetters, JbÖB 15, 1966, 273; Sande (Anm. 14) 85 ff. Abb. 26. 27; Foss (Anm. 49) 65 mit Anm. 38; 67 mit Anm. 43 Abb. 21; İnan - Alföldi-Rosenbaum (1979) 27. 30 f. 160 Nr. 112; Foss (Anm. 48) 196 ff. 204. 208 f.; Goette (1990) 63. 147 E 13 Taf. 45, 6; Bauer (Anm. 14) 283. 296. 425; Horster a. O. 46; Feissel (Anm. 48 ) 91 ff.; dazugehörige Basisinschrift: IvE 1310.

69 s. o. Anm. 68.

70 Konträr dazu eine Statue aus Ephesos im Museum von Izmir: s. Kollwitz (1941) 87 f. Nr. 10 Taf. 31, 1; Oberleitner (1959) 88. 97; Foss (Anm. 48) 196 f. mit Anm. 2. 5; Özgan - Stutzinger (1985) 254; Goette (1990) 146 E7 Taf. 45, 1; Bauer (Anm. 14) 283. 423. 
in Athen ${ }^{71}$ zu bestehen, beide Büsten sind durch Details in der Gestaltung der Falten verbunden. Zum einen ist es die unruhige Anordnung der vertikalen Falten unter dem Umbo, zum anderen die sorgfältige Ausarbeitung des Colobium ${ }^{72}$ mit ähnlichem Verlauf der Falten - besonders der Bogenfalte über der rechten Brust. Auch zieht bei der Büste in Athen der Umbo mit seinem unteren Abschluß leicht schräg über die Brust.

Es ist schwierig, die Büste mit Büstenfuß aus Ephesos ${ }^{73}$ für den Vergleich mit der Büste aus Balçova heranzuziehen, da sie unfertig und sehr 'handwerklich' ausgeführt ist - obwohl sie, speziell den Verlauf und die gesamte Gestaltung des Umbo betreffend, unserer Büste sehr nahesteht.

Da man Togabüsten auch als Teil von Ganzstatuen betrachten kann ${ }^{74}$, erscheint ein Vergleich dieser beiden Denkmälergattungen legitim. Wie bereits ausgeführt, entspricht die Toga der Büste aus Balçova dem Typus $E^{75}$ nach H. R. Goette. Der voll ausgebildete Typus E ist für Goette »... einige Jahre später, seit Beginn des 5. Jahrhunderts - sicher datierbar durch die Basis des Theodosius-Obelisken ${ }^{76}$ in Istanbul,$- \ldots$ voll ausgeprägt nachweisbar. $\ll^{77}$ In der Rundplastik sieht Goette zwei Statuen in Rom ${ }^{78}$ als die ersten statuarischen Wiedergaben seines Typus E an. Die spätesten durch Basen mit Inschrift datierbaren Togastatuen sind 'Pytheas' ${ }^{\text {'9 }}$ und 'Palmatus' ${ }^{80}$, beide aus Aphrodisias. Sie werden an das Ende des 5. bzw. an den Beginn des 6. Jahrhunderts datier $^{81}$. Innerhalb der Zeitspanne vom Ende des 4. bis an den Beginn des 6. Jahrhunderts muß auch unsere Büste angesiedelt werden.

Immer wieder stößt man bei der Datierung spätantiker Porträtstatuen auf das grundlegende Problem, daß eine Statue selten durch äußere Umstände datiert werden kann: so ist aus Ephesos kein einziges außerstilistisch datiertes Beispiel bekannt ${ }^{82}$. In Aphrodisias konnten die in Sturzlage gefundenen Statuen - manche mit Porträtkopf - teilweise mit den namengebenden Inschriften auf den Basen in Verbindung gebracht werden ${ }^{83}$.

Was die Entwicklung der Togadarstellung betrifft - ausgehend von den datierten Monumenten -, so dürfte die Stilentwicklung, grob gesagt, in einer graduellen Abnahme der Plastizität verlaufen. In der Detailbehandlung der Togafalten der Statuen bedeutet dies eine Abnahme der

71 s. o. Anm. 57.

$72 \mathrm{Zu}$ dem Untergewand, dem Colobium, s. Delbrueck (Anm. 9) 33. $43 \mathrm{f}$.

73 s. o. Anm. 66.

74 Dies gilt nicht für die zwei aus Ephesos stammenden, äußerst qualitätvollen Büsten (s. o. Anm. 62). Sie haben beide die vertikale Faltenpartie unterhalb des Umbo bis über die rechte Brustseite gezogen, bei einer der Büsten ist ganz rechts außen noch eine Bogenfaltenreihe angedeutet. Wären sie nur eine verkürzte Wiedergabe der Toga, hätte man sich ab der Brustmitte auf der rechten Seite Bogenfalten unterhalb des Umbo zu erwarten, wie wir es bei allen statuarischen Vertretern der Togati finden. Die aus einer Statue umgearbeitete Büste aus Ephesos (s. o. Anm. 61) weist auch nur vertikale Falten auf. Bei den beiden 'handwerklich' gearbeiteten Büsten aus Ephesos (s. o. Anm. 67) reicht der untere Abschluß des Umbo an der rechten Brustseite bis an den Büstenfuß.

75 Goette (1990) $62 \mathrm{f}$.

76 G. Bruns, Der Obelisk und seine Basis auf dem Hippodrom von Konstantinopel, IstForsch 7 (1935); H. Kähler, Philologische Wochenschrift 59, 1939, 93 ff.; J. Kollwitz, Gnomon 13, 1937, 423 ff.; E. Weigand, ByzZ 37, 1937, 452 ff.; St. Rebenich, IstMitt 41, 1991, 447 ff.; zuletzt: B. Kiilerich, The obelisk base in Constantinople: Courtart and imperial ideology, ActaAArtHist 10, 1998, 5 ff.

77 Goette (1990) 62.

78 Rom, Konservatorenpalast Inv. 896: s. Goette (1990) 62 f. E8 Taf. 46, 1 (mit älterer Lit.). - Rom, Konservatorenpalast Inv. 895: s. Goette (1990) 62 f. E9 Taf. 46, 2 (mit älterer Lit.).

79 Zuletzt Smith (Anm. 14) 167 f. Abb. 8 Taf. 4.

80 Zuletzt Smith (Anm. 14) 168 Abb. 9 Taf. 3.

81 Inschrift der Pytheas-Basis: C. Roueché, Aphrodisias in Late Antiquity (1989) 93 Nr. 56 Taf. 13. 14; Inschrift der Palmatus-Basis: ebenda 102 f. Nr. 62 Taf. 16.

82 An dieser Stelle soll auf diese Problematik nicht näher eingegangen werden. Die spätantiken Porträtstatuen sind für den Corpus der ephesischen Plastik in Vorbereitung, M. Laubenberger (Wien) hat zur Bearbeitung die spätantiken Porträts und Büsten übernommen. Die Verf. beschäftigte sich eingehend mit spätantiken Porträtstatuen, besonders mit Togadarstellungen: J. Auinger, Chronologieprobleme spätantiker Porträtstatuen. Die Gruppe der Togati (ungedr. Mag. Wien 1999).

83 Smith (Anm. 14) 155 ff. 
naturalistischen Darstellung hin zu einer immer starreren Wiedergabe des Stoffes. J. Kollwitz ${ }^{84}$ sieht die Statuen nur mehr zweidimensional in die Ebene geklappt: Sie seien am Ende ihrer Entwicklung nicht mehr als dreidimensionale Gebilde aufzufassen, sondern in eine Ebene projiziert und stark frontal ausgerichtet ${ }^{85}$.

Obwohl 'Arcadius' ${ }^{86}$ und 'Valentinian'87 aus Aphrodisias nicht dem Typus E nach Goette entsprechen ${ }^{88}$, sollen sie hier erwähnt werden, da sie Elemente an der Bekleidung aufweisen, die gerade auch an unserer Büste wiederzufinden sind. Ihre Entstehung ist zwischen 388 und 392 anzusetzen $^{89}$, sie sind somit als die frühesten Beispiele spätantiker Togastatuen in Kleinasien anzusehen.

Als die frühesten statuarischen Exemplare des Typus E sind die oben erwähnten, durch ihre Porträts datierten Statuen im Westen des Reiches, in Rom, zu nennen ${ }^{90}$. Die beiden Statuen wurden 1879 in Rom in einer mittelalterlichen Mauer verbaut gefunden. Die Gewandangabe folgt ganz klar den Körperkonturen, der Stoff des Gewandes scheint organisch über den Körper gelegt. Bei der Toga ist der breite, aufgefächerte Umbo auffällig, der breit unter dem rechten Arm herausgezogen und nicht ganz horizontal über die Brust gelegt ist, sondern in leicht geschwungenem Bogen in Richtung der linken Schulterpartie zieht. Die Statuen werden in der Forschung fast ausnahmslos um 400 datiert $^{91}$.

'Palmatus' und 'Pytheas' sind zwei Statuen ${ }^{92}$, die mit Porträtköpfen erhalten sind. Die Gewandung beider Statuen zeichnet sich durch ein starres Faltenschema aus: so finden sich unter dem Umbo auf der rechten Seite Bogenfalten, die auf der linken Seite von vertikalen Faltenbahnen abgeschnitten werden. Zumeist treten drei breite Faltenbahnen mit unterschiedlicher Binnenzeichnung auf, bei Pytheas sind es z. B. langgezogene, tropfenförmige Falten, die in die Oberfläche eingetieft sind.

Beginnend mit den datierten Exemplaren um 400 bis zu den Statuen des 'Palmatus' und 'Pytheas' aus Aphrodisias ist in der Forschung anhand stilistischer Kriterien der Großteil der Statuen chronologisch gereiht worden. Wegen kontroverser Ansichten zur stilistischen Entwicklung soll die erstellte Chronologie hier aber nicht näher ausgeführt werden.

Nachdem die Stilentwicklung der Toga anhand der außerstilistisch datierbaren Statuen kurz gezeigt wurde, soll eine Zuordnung der Gewandung der Büste aus Balçova versucht werden. Ausschlaggebend für eine zeitliche Fixierung scheinen die Faltenpartien unterhalb des Umbo zu sein. An der rechten Brustseite finden sich unterhalb des Umbobeginns Bogenfalten - dieses Motiv verbindet alle statuarischen Togadarstellungen. Danach folgen unruhig gehaltene Vertikalfalten, die bei späteren statuarischen Togadarstellungen einem strengen Schema unterliegen ${ }^{93}$. Genau diese Vertikalfalten verbinden augenfällig unsere Büste mit frühen Darstellungen der spätantiken Toga; wir kennen sie bereits an 'Arcadius' und 'Valentinian' aus Aphrodisias ${ }^{94}$. Auch

84 Kollwitz (1941) 95.

85 Dieser groben Entwicklungslinie folgte die Forschung: Kollwitz (1941) 94 ff.; Oberleitner (1959; 1964/65); Özgan - Stutzinger (1985) 248 ff.; Goette (1990) 63 (ohne Versuch einer Feindatierung). In der Forschung wurden bisher jedoch nicht die verschiedenen Provenienzen der Statuen bedacht.

86 Zuletzt Smith (Anm. 14) 162 Abb. 4 Taf. 12, 1-2.

87 Smith (Anm. 14) 162 Abb. 3 Taf. 1, 1.

88 s. o. Anm. 10.

89 s. Roueché (Anm. 81) 47 ff. Nr. 25-27 Taf. 7.

90 s. o. Anm. 78.

91 Anders: Oberleitner (1959) 95; Oberleitner (1964/65) 13. W. Oberleitner will die beiden Togati in konstantinische Zeit setzen, vor allem durch den Vergleich mit den Postamentreliefs des Konstantinsbogen. Jedoch ist m. E. zu bedenken, daß demnach die beiden Togati aus Rom die frühesten statuarischen Belege für den Typus E der Toga wären, obwohl auf offiziellen Reliefs der Zeit die Toga im Typus D - jene mit vollcontabuliertem Umbo - dargestellt ist.

92 s. o. Anm. $79-81$.

93 s. dazu die beiden Statuen des 'Pytheas' und 'Palmatus' aus Aphrodisias.

94 s. o. Anm. 86. 87. 
die Falten des Umbo selbst sind vergleichsweise naturalistisch gebildet. Die an der schmalsten Stelle des Umbo fächerförmig ausgebreiteten Falten überschneiden einander und sind von unterschiedlicher Breite und Tiefe. In lebendigem Spiel, wie wir es von den beiden Statuen aus Rom ${ }^{95}$ kennen, ziehen sie über den Brustkorb. Der Gesamteindruck der Büste aus Balçova ist daher m. E. gut mit den beiden Statuen aus Rom vergleichbar. Auch hier unterliegen die Faltenpartien unterhalb des Umbo noch keinem starren Schematismus, sondern sind lebendig gestaltet.

So könnte die Darstellung der Toga an unserer Büste im Vergleich mit den wenigen sicher datierten Exemplaren an eine etwas frühere Datierung denken lassen, nämlich an den Beginn des 5. Jahrhunderts n. Chr. Dieser Ansatz muß vorerst hypothetisch bleiben, da die Zeitspannen der typologischen Entwicklung der Toga noch nicht sicher bekannt sind. Der frühe zeitliche Ansatz kann lediglich für die Büste aus Balçova angenommen werden, da wir nicht wissen, wie lange die Werkstatt der Eutropius-Gruppe tätig war. Das hier vorgestellte Bildwerk fügt jedenfalls der Eutropius-Gruppe ein weiteres Mitglied hinzu ${ }^{96}$, das auf Grund der erhaltenen Büste erstmals einen Gesamteindruck dieser Denkmäler bietet. Die leicht vorgestreckte Haltung des Kopfes gegenüber der senkrechten Büste erinnert an die 'shield portraits' aus Aphrodisias ${ }^{97}$.

Mag. Johanna Auinger

Obere Amtshausgasse 45/3, A-1050 Wien

E-Mail:j_auinger@hotmail.com

Abbildungsnachweis: Abb. 1-8: Izmir, Arkeoloji Müzesi Inv. 5246, Photo N. Gail; alle Abb. C ÖAI.

95 s. o. Anm. 78.

96 Ein weiteres Fragment, das m. E. der Eutropius-Gruppe zuzuordnen ist, wurde im Sommer 2002 bei den Grabungen im Vediusgymnasium gefunden. Zu den neuen Grabungen s. vorläufig: M. Steskal - M. La Torre, ÖJh 70, 2001, 221 ff. Da der Verf. eine persönliche Autopsie noch nicht möglich war, kann es im Rahmen dieses Beitrags leider nicht vorgestellt werden.

97 s. R. R. R. Smith, JRS 80, 1990, 127 ff.; ders., RM 102, 1995, 331 ff. 